\title{
Mapping diabetes research in Middle Eastern countries during 2007-2013: A scientometric analysis
}

\author{
Zahra Emami ${ }^{1}$, Nadjla Hariri ${ }^{{ }^{*}}$, Mohammad Ebrahim Khamseh ${ }^{2}$, Fatemeh Nooshinfard ${ }^{1}$ \\ Received: 3 May 2016 \\ Published: 10 Sep 2018
}

\begin{abstract}
Background: Scientometric studies are highly important, as they provide information about scientific products worldwide and empower scientists to compare research activities in different regions. The present study was conducted to map scientific research in diabetes mellitus using scientometric analysis in Middle Eastern countries during 2003- 2007.

Methods: This was an analytical study with a scientometric approach. The study population was formed by the indexed scientific results of diabetes mellitus in the Web of Science database during 2007-2013. Data were analyzed using Excel, and HistCite to map the scientific texts.

Results: A total of 6532 records were retrieved from 3926 institutions. These records belonged to 19323 authors and were published in 1420 journals.

The Journal of Diabetes Care, with 3928 citations, had the most global citation score (GCS). The Journal of Diabetes Research and Clinical Practice, with 185 citations, achieved the first degree of local citation score (LCS). Most of the scientific documents produced in the Middle East belonged to Turkey (31.91\%) and Iran (21.7\%). Seven scientific clusters based on LCS, and 5 based on GCS existed in the scientific mapping. Topical clusters based on global and local indices showed that the prevalence of diabetes, hyperglycemia, and pregnancy outcomes, diabetic risk factors, diabetic complications and their new treatments, and glucose monitoring in Type 1 diabetes were the fields being addressed in the main articles of the clusters.

Conclusion: Scientific production and local and global citations in diabetes research in Iran $(21.7 \%$ of diabetes research in the Middle East) have elevated the country to a prominent position. Top ranking countries in diabetic research were Turkey, Iran, and Israel, respectively. Moreover, this paper quantified the studies that were done on different aspects of diabetes. The results of this study can be used by health care providers to employ the best multidisciplinary approach for managing diabetes and its complications. Also, the results can help the policy-makers and governments to determine the priorities for budget allocation based on the burden of diseases. Establishing a regional diabetes network in the Middle East can be beneficial and lead to scientific collaboration and an increase in scientific production in this field.
\end{abstract}

Keywords: Scientific research, Diabetes mellitus, Middle East, Scientometric

Copyright $₫$ Iran University of Medical Sciences

Cite this article as: Emami Z, Hariri N, Khamseh ME, Nooshinfard F. Mapping diabetes research in Middle-Eastern countries (during 2007and 2013): A scientometric analysis. Med J Islam Repub Iran. 2018 (10 Sep);32:84. https://doi.org/10.14196/mjiri.32.84

\section{Introduction}

Diabetes mellitus, a leading cause of death and disability $(1,2)$, involves 387 million people worldwide; this number will rise to 592 million by the year 2035 (3). The worldwide prevalence of diabetes was about $8 \%$ in

\footnotetext{
Corresponding author:Dr Nadjla Hariri, nadjlahariri@gmail.com
}

1. Department of Knowledge \& Information Science, Science and Research Branch, Islamic Azad University, Tehran, Iran.

2. Endocrine Research Center, Institute of Endocrinology and Metabolism, Iran University of Medical Sciences, Tehran, Iran.
2011 and is expected to rise to $10 \%$ by the year 2030 (4). Of diabetic patients, $77 \%$ live in low- and middle-income countries; $10 \%$ of adults in the Middle East and North Africa have diabetes (3).

$\uparrow$ What is "already known" in this topic:

Scientific production in the field of diabetes shows an increasing trend in almost all countries. In addition, diabetes research consists of the majority of scientific productions in the field of endocrinology because of the high prevalence of this disease.

$\rightarrow$ What this article adds:

In this study, an ascending trend of research in diabetes was found in the Middle East including Iran. The results of this study can be used to set priorities for allocation of the budget and development of preventive and therapeutic programs in the field of diabetes. 
Published articles in the scientific journals are the main communication tools in different fields of science, and scientometric analysis is one of the most common evaluation methods of scientific activities and research management.

The indexed scientific products in the Web of Science are among the most important indices that show the success of each country in publishing their scientific findings at the international level. More than 2 million articles are published in the field of medicine annually (5).

The goals of scientific studies are to evaluate scientific products and their impact on different fields, researchers, universities, and countries (6).

In addition, international scientometric studies are of significant importance because they provide worldwide scientific competition, leading to development of scientific products in different countries and shifting them towards the world standards of scientific production (7).

There are several studies in the field of scientometrics and scientific products. Escudero et al. evaluated scientific products of Spain in the field of rare diseases during 19992000. They found 2978 documents $(82.5 \%$ original articles and $17.3 \%$ reviews) in this field. Their results indicated the small number of research in the field of these rare diseases (8). Vitzthum et al. evaluated scientific production of scoliosis (density-equalizing) during 19072007. Their results revealed that 8186 documents from 76 countries had been published in this field. The USA, UK, and Canada had the largest number of published articles, and Bradford produced the largest amount of records (146 records) (9).

In 2010, Bala and Gupta evaluated the scientific mapping of neuroscience during 1999- 2008. They found that India produced $0.99 \%$ of the world's products in neuroscience. Also, their results indicated an urgent need for increasing research activities in this field (10). Li et al. conducted a study "Global stem cell research trend: Bibliometric analysis as a tool for mapping the trend from 1991 to 2006"; and they found that the scientific products in this field, based on Web of Science, included 2493 journals and 163 specific topics. A large number of studies were in the fields of hematology, oncology, and biology. Seven industrial countries were the top scientific producers in this field (11). In 2007, Patra assessed the rate of science production in the field of HIV/AIDS in India. The results revealed that during 1982-2005, a total of 5071 authors compiled 2178 papers related to AIDS. The most active author was Solomon from The Madras Medical College and Government General Hospital. Indian researchers published 118 papers in the Indian Journal of Medical Research. The University of Delhi, with 528 articles $(24.2 \%)$, achieved the first ranking of science production among universities and institutions. This study showed that Indian productions in this field are still low at the national level (12). Yang et al. evaluated articles in the field of pediatric dentistry. They suggested that there is a need for a computer system to facilitate the access of clinically useful literature (13). Ramos et al. showed that scientific products in the field of microbiology have been increased in Spain during 1990-
2002 (14). Moreover, Signore et al. evaluated the articles in the field of nuclear medicine. Among the European countries, Germany has made the greatest contribution to the production of articles in nuclear medicine (15). Stegmann and Grohmann assessed the articles related to bird flu. They suggested that an international cooperation is necessary between Europe and Asia (16). Krishnamoorthy et al. showed a significant research activity in diabetes mellitus using the bibliometric analysis of the literature on diabetes (17). Also, Arunachalam and Gunasekaran (18) assessed the diabetes research in India and China.

In Iran, Peykari et al. assessed diabetes research in the Middle East. They concluded that more collaboration between researchers is needed to maintain the increasing trend of diabetes (19). Peymani et al. evaluated Iranian stem cell research trends and indicated that although there was an increasing trend in scientific productions in this field, the rate of citations was still low (20). Shahram et al. evaluated the scientometric analysis and mapping of scientific articles on Behcet's disease (21).

Furthermore, Iranian researchers in the field of scientometry work on different aspects of medical science, such as Hamdipour and Osareh (22) in the field of MS, Shirshahi (23) in surgery, Hodhodinejad (24) in traditional medicine, Namdar (25) in parasitology, Janmohammadi (26) in Orthopaedic, and Makizadeh in medical ethics (27).

Nowadays, researchers use software for relational bibliometric analysis, while, in the past, simple hand drawn diagram of citations between authors, journals, or articles were used for this purpose. Recently, researchers have developed a software that performs this process automatically (28).

For example, HistCite ${ }^{\mathrm{TM}}$ software, produced by Garfield, visualizes citations between a group of articles based on a period of time (29). This software, histCite ${ }^{\mathrm{TM}}$, facilitates the understanding of paradigms through identifying the most important works in a given topical field, while providing graphics and presenting them based on priority of citation links at the same time (29). The input of this software is bibliographic records and their citations that are extracted from Web of Science (WoS) or other sources. The output is tables or graphics with informetric for data $(30,31)$.

It is possible to visualize most related articles among the collection of retrieved documents using histCite ${ }^{\mathrm{TM}}$ (32).

To use HistCite ${ }^{\mathrm{TM}}$ software in mapping the histography of scientific products, Luico-Arias and Leydesdroff searched 2 terms "fullerene*" and "nanotube" as indexed documents in the Web of Science and retrieved 9672 and 7996 documents, respectively. They found 30 highcitation documents in each group and their internal relations and visualized them by histCite software. They tried to validate more results of histography with histCite software using the theory of information and analysis of social networks (32).

According to the study of Guz and Rushchitsky, Web of Science and Scopus are often used to search the literature in various fields of science. WoS from Thomson Reuters 
(ISI) was the only citation database and publication that has been covering all domains of science for many years (33).

Thus, considering the importance of diabetes as a common disease of endocrinology and metabolism, conducting this research seemed necessary to evaluate the scientific products of this disease in Iran and compare it to that of other countries in the Middle East.

Comparison of the existing situation in Iran to other countries of the region, with respect to scientific products, will help the policy-makers to provide the optimum plans and services. Therefore, the aim of this study was to map scientific products in the field of diabetes in Iran and the Middle East by searching the Web of Science during 2007-2013.

\section{Methods}

This was an analytic research with a scientometric approach. The study population was selected using the indexed scientific results of diabetes mellitus in the Web of Science during 2007-2013. Data collection was performed by searching the advanced search part of WoS. To identify and extract scientific products in this field, the following search strategy was done in the advanced search part of the WoS.

Descriptors and keywords were used to retrieve the articles. Initially, the keyword "diabetes" was searched in the Medical Subject Headings (MeSH) and used as an entry term. Subheadings of the entry term, which were near to the descriptor and referral keywords, were selected for searching. Each of the descriptors searched in the title were retrieved. According to the Alexander (34) study, during a simultaneous search in the title, abstract, and keywords of the articles, the retrieved records were not relevant. Thus, it was preferred to search for more keywords and descriptors in a specific field. The studies showed that $90 \%$ of the related results could be retrieved using this method. In the next step, the results of the search were limited to Middle Eastern countries (Iran, Iraq, Turkey, Egypt, Bahrain, Jordan, Kuwait, Lebanon,
Oman, Qatar, Saudi Arabia, Syria, United Arab Emirates, Cyprus, Israel, and Palestine) (35) and time (2007-2013).

TI $($ Title $)=($ Descriptor or Entry Term) AND CU $($ Country $)=($ Country Name $)$ AND Publication Year $=$ (2007-2013)

\section{Data Source and Sample (Data Collection)}

Web of Science database (Thomson Reuters) was used as the only source of data, and all papers retrieved during 2007-2013 were evaluated. MeSH (Medical Subject Heading) of Medline was used and the descriptors and entry terms related to diabetes mellitus were selected.

1) Inputting the keyword "diabetes mellitus" in $\mathrm{MeSH}$ major field [majr] and

2) Searching a subheading and entry term of diabetes mellitus

\section{Keywords}

The key terms were as follow:

Title $(\mathrm{TI})=$ Keywords AND Publication Date (DP) 2007-2013 AND Country $(C U)=$ Middle East

Descriptors:

Diabetes Complications $(\mathrm{N}=11$ subheading $) \quad(\mathrm{N}=34$ entry term) $(\mathrm{N}=1$ see also $)$ total $=46$

Diabetes, Gestational ( $\mathrm{N}=3$ entry term) total keyword $=46$

Diabetes Mellitus, Experimental $=(\mathrm{N}=4$ entry term $)$ total $=4$

Diabetes Mellitus, Type $1=(\mathrm{N}=1$ subheading $)(\mathrm{N}=19$ entry term) total $=20$

Diabetes Mellitus, Type $2=(\mathrm{N}=1$ subheading $)(\mathrm{N}=18$ entry term $)(\mathrm{N}=1$ see also $)$ total $=19$

Donohue Syndrome $=(\mathrm{N}=4 \mathrm{entry}$ term $)(\mathrm{N}=2$ see also $)$ total $=6$

Prediabetic State $=(\mathrm{N}=1$ entry term $)(\mathrm{N}=1$ see also $)$ total keyword $=2$

The data extracted from WoS were saved as files containing 500 documents in the form of plain text and all were merged. Then, they were analyzed with co-

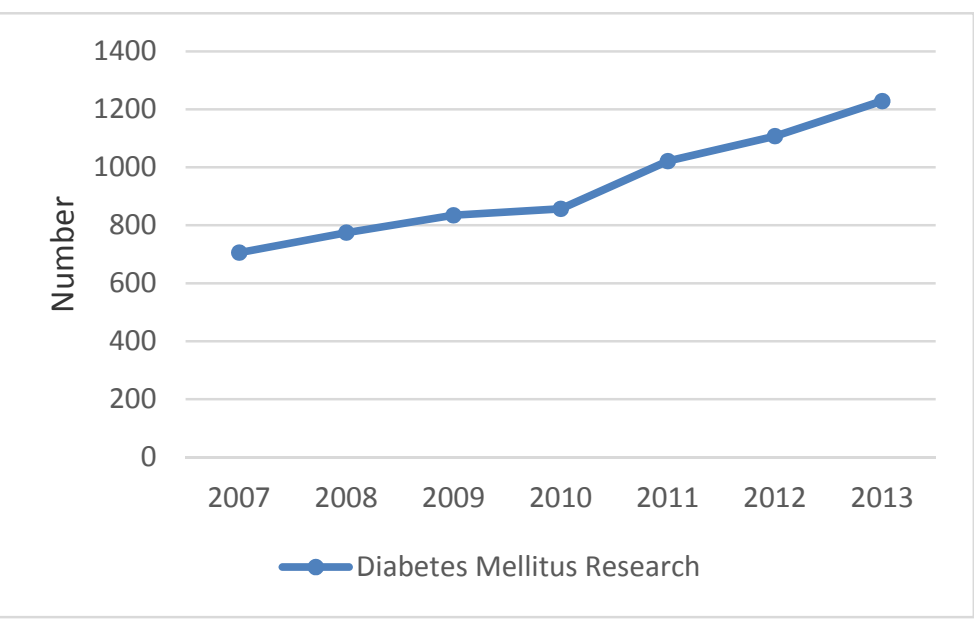

Fig. 1. Trend of scientific production in diabetes in the Middle East during 2007-2013 
author.exe. This software was designed to analyze the data of the citation index of the science. Additionally, it was in accordance with the used methods in the field of organization and arrangement of the existing data in this database. Co-author.exe software found, analyzed, and counted the data and allowed the excel software to arrange and analyze them.

In the second stage, the extracted data were transferred from WoS to HistCite software. Then, the essential analyses were performed and scientific maps were visualized based on the most highly cited articles. In this software, the mapping of histography can be performed based on 2 indexes: LCS (Local Citation Score) and GCS (Global Citation Index).

LCS shows the number of citations of a document to the software, and GCS is the frequency with which one document, author, or institution is cited by all the documents in WoS, including LCS.

\section{Results}

With a thorough search in the WoS, 6532 records were retrieved in the field of diabetes during 2007-2013.

Figure 1 displays the trend of scientific productions in diabetes mellitus in the Middle East during 2007-2013. The highest output was related to 2013 (1229 documents), the highest number of global citations belonged to 2007 (10 535 citations), and the least citations to 2013 (5095 citations). The highest and lowest number of local citations belonged to 2008 (773 citations) and 2013 (46 citations), respectively. During this time (2007-2013), the trend of scientific production was increasing.
Based on TLCS and TGCS indices, the highest and lowest number of local citations belonged to 2008 (773 citations) and 2013 (46 citations), respectively. Global citations were as follow: 2007 with 10535 citations, 2008 with 9980 citations, 2010 with 8318 citations, 2009 with 8171 citations, 2011 with 6471 citations, 2012 with 6387 citations, and 2013 with 5095 citations.

According to the number of publications, Turkey, with 2126 scientific documents on diabetes, was ranked first among Middle Eastern countries during the studied period and Iran, with 1446 scientific documents (21.7\%), was ranked second. Syria and Yemen had the least number of documents, 8 and 6 scientific documents $(12 \%, 0.09 \%)$, respectively. There were no documents from the Palestinian territories. Based on TGCS, Israel (with 17 579 documents) and Turkey (with 14591 documents) had the highest number of global citations. The first and second ranks of local citations belonged to Turkey, with 1022 documents, and Iran, with 812 documents (Table 1).

With respect to the authors who had the most scientific productions and were most cited in the field of diabetes in the WoS during 2007-2013, it was determined that in the Middle East, 19323 authors worked in the field of outputs of diabetes.

Determining important journals in each field of science is of prime importance. One of the important criteria in the evaluation of journals is the amount of citations of the papers of that journal. In the retrieved documents on diabetes, it was found that the papers were published in 1420 journals. The Diabetes Research and Clinical Practice, with 144 papers, and Diabetologia, with 143

Table 1. Top ranking Middle Eastern Countries in diabetes research during 2007-2013

\begin{tabular}{lcccc}
\hline Country & Document & TLCS & TGCS & Total \% \\
\hline Turkey & 2126 & 1022 & 14591 & 31.9 \\
Iran & 1446 & 812 & 9678 & 21.8 \\
Israel & 1104 & 613 & 17579 & 16.6 \\
Egypt & 607 & 169 & 3756 & 9.1 \\
Saudi Arabia & 547 & 160 & 3991 & 8.2 \\
UAE & 210 & 133 & 1884 & 3.1 \\
Kuwait & 174 & 74 & 1330 & 2.6 \\
Jordan & 96 & 48 & 775 & 1.4 \\
Lebanon & 88 & 41 & 865 & 1.3 \\
Qatar & 64 & 29 & 540 & \\
Bahrain & 62 & 57 & 565 & 0.9 \\
Oman & 50 & 13 & 340 & 0.8 \\
Iraq & 49 & 9 & 781 & 0.7 \\
Cyprus & 25 & 3 & 77 & 0.4 \\
Syria & 8 & 5 & 31 & 0.1 \\
Yemen & 6 & 3189 & 56260 & 1 \\
Total & 6662 & & & \\
\hline
\end{tabular}

TGCS: Total Global Citation Score

\begin{tabular}{|c|c|c|c|c|c|}
\hline Number & Journal & Recs & Total $\%$ & TLCS & TGCS \\
\hline 2 & Diabetologia & 143 & 10.07 & 26 & 408 \\
\hline 4 & Diabetes Care & 89 & 6.26 & 161 & 3928 \\
\hline 5 & Journal of Diabetes and Its Complications & 77 & 5.42 & 97 & 1012 \\
\hline 6 & Clinical Biochemistry & 71 & 5 & 18 & 289 \\
\hline 9 & Acta Diabetologia & 52 & 3.66 & 28 & 451 \\
\hline 10 & Annals of Nutrition and Metabolism & 52 & 3.66 & 1 & 85 \\
\hline
\end{tabular}




\begin{tabular}{clcccc}
\multicolumn{2}{c}{ Table 3. Top ranking universities and academic institutions in diabetes research in Middle Eastern countries } \\
\hline Number & Institution & Recs & Total \% & TLCS & TGCS \\
\hline 1 & Tehran Univ Med Sci & 417 & 10.62 & 295 & 3352 \\
2 & Tel Aviv Univ & 359 & 9.14 & 253 & 6713 \\
3 & King Saud Univ & 243 & 6.18 & 58 & 1838 \\
4 & Istanbul Univ & 217 & 5.52 & 105 & 2041 \\
5 & Isfahan Univ Med Sci & 180 & 4.58 & 107 & 1628 \\
6 & Technion Israel InstTechnol & 152 & 3.87 & 130 & 1913 \\
7 & Cairo Univ & 137 & 3.48 & 39 & 712 \\
8 & Gazi Univ & 130 & 3.31 & 84 & 1046 \\
9 & Hebrew Univ Jerusalem & 129 & 3.28 & 72 & 2479 \\
10 & Kuwait Univ & 127 & 3.23 & 73 & 1108 \\
\hline
\end{tabular}

papers, achieved the first and second ranks, respectively. Based on citation index, the Journal of Diabetes Care, with 3928 citations, had the highest number of global citations and New England Journal of Medicine, with 2215 citations, and Diabetes Research and Clinical Practice, with 1525 citations, were in the next ranks, respectively (Table 2).

The first 3 journals with local citations were Diabetes (185 citations), Diabetes Care (161 citations), and Journal of Diabetes and its complications (97 citations).

With respect to universities and institutions that produce science in the field of diabetes, the retrieved records showed that 3926 institutions were active. In this field, the highest number of retrieved records belonged to Tehran University of Medical Sciences (417 documents) and Tel Aviv University (359 documents). In general, universities had the most important role in the production of publications in the field of diabetes (Table 3).

With regards to the format, articles achieved the highest rank (4459 records), followed by meeting abstracts (1351 records), letters (235 records), reviews (190 records), article proceeding papers (151 records), and editorial material (97 records).

It was found that most publications in the field of diabetes in the Middle East were published in English (646 documents) and other languages, such as Turkish (51 documents), French (11 document), Arabic (2 documents), German, and Spanish, were in the next ranks.

Map of science makes spatial representation of the relationship among fields, specialists, and individual papers, and authors. They are similar to geographic maps which show the physical relations on earth. The cited networks shown in this spatial map reveal the patterns of relationship and scientific collaborations as well as the processes of the local and global citations of the researchers.

In this study, HistCite software was used for scientific mapping. In this mapping, the LCS index refers to the citations that are received among the survived documents, and GCS index refers to the citations that the evaluated records have received from all indexed documents in the WoS. To do the mapping, among 6532 retrieved documents, 250 documents were selected based on world and local citations, then, the performed clusters were assessed. In the world citation, 250 top documents in this field had the maximum of 1076 and minimum of 3 citations. In the local citation, 250 top documents had the maximum of 46 and minimum of 3 citations.
Figure 2 demonstrates the mapping of diabetes mellitus based on LCS index during 2007-2013. In this map, the circles with the higher dimension show the highest rate of citations. This map consists of 7 clusters.

The first cluster was formed during 2007-2011 and consisted of 11 articles. In this cluster, the highest rate of local and global citations belonged to the article of Metzger BE, et al., with 43 local and 1076 global citations. This article, published in NEJM in 2008, is about hyperglycemia and adverse pregnancy outcomes.

The second cluster included 5 articles that were conducted in 2008. This cluster was related to collaboration of Iranian authors; the main subject of this cluster was different aspects of diabetic foot ulcer. The largest number of local and global citations was 7 and 15, respectively.

The third cluster was formed during 2007-2010 and consisted of 6 articles. In this cluster, the highest rate of local and global citations belonged to the article of Onat A, et al. (GCS=93, LCS=19). This article "Determinants and definition of abdominal obesity related to the risk of diabetes, metabolic syndrome, and coronary disease in Turkish males: A prospective cohort study" was published in Atherosclerosis in 2007.

The forth cluster was formed during 2007-2010 and consisted of 7 articles. The central and high- citation record of this cluster was the record number 910 written by an Israeli author named Milman U, (LCS $=16$, $\mathrm{GCS}=126$ ). All these articles were the results of collaboration of Israeli authors.

The fifth cluster was formed with 5 articles during 2007-2012. This cluster consisted of Iranian and Israeli authors. The record number 540 had the highest local (9) and global (6) citations. The most important article in this cluster belonged to Parisa Sarkheil from Iran (Antidiabetic effect of Phlomis anisodonta: Effects on hepatic cells lipid peroxidation and antioxidant enzymes in experimental diabetes).

The sixth cluster consisted of 6 records, and the most important record (record number 560) in this cluster was an article by Masoud Soheilian, with 12 local and 60 world citations. The topic of this article was as follows: Intravitreal bevacizumab (Avastin) injection alone or combined with triamcinolone versus macular photocoagulation as a primary treatment of diabetic macular edema.

The seventh cluster was formed with 5 articles during 2007-2009. This cluster was the result of collaboration of 


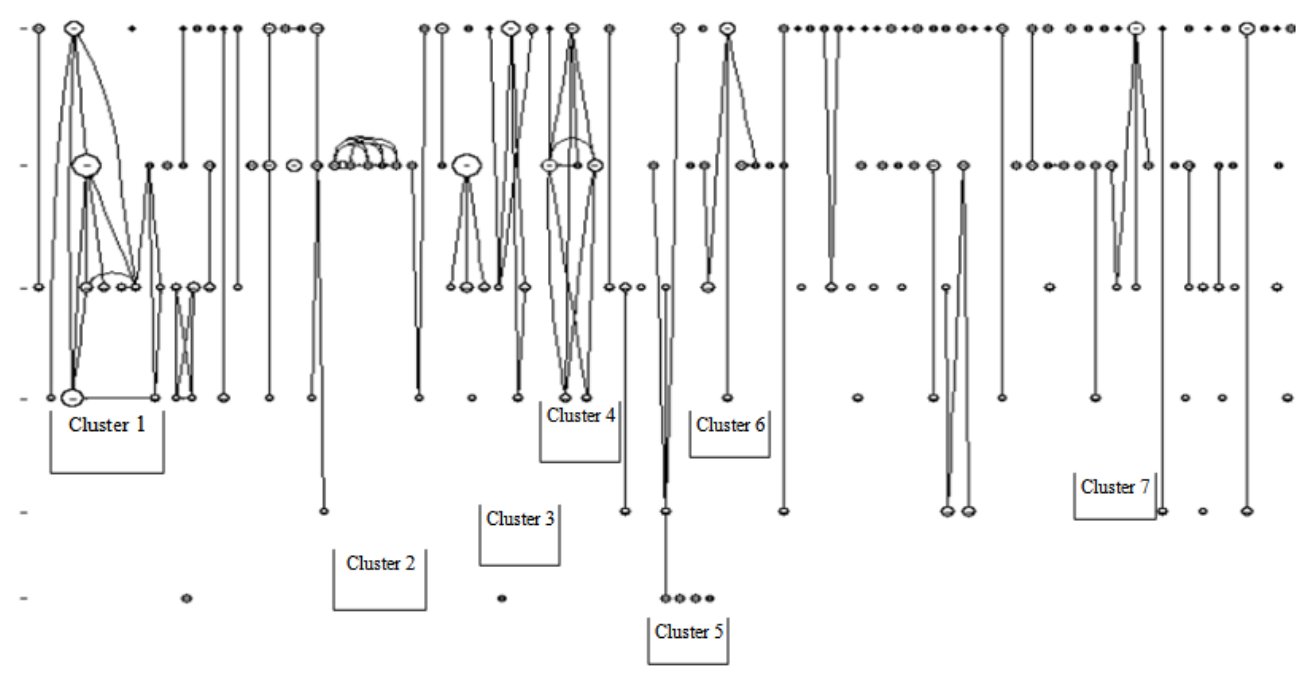

Fig. 2. Mapping of diabetes mellitus based on LCS index during 2007-2013

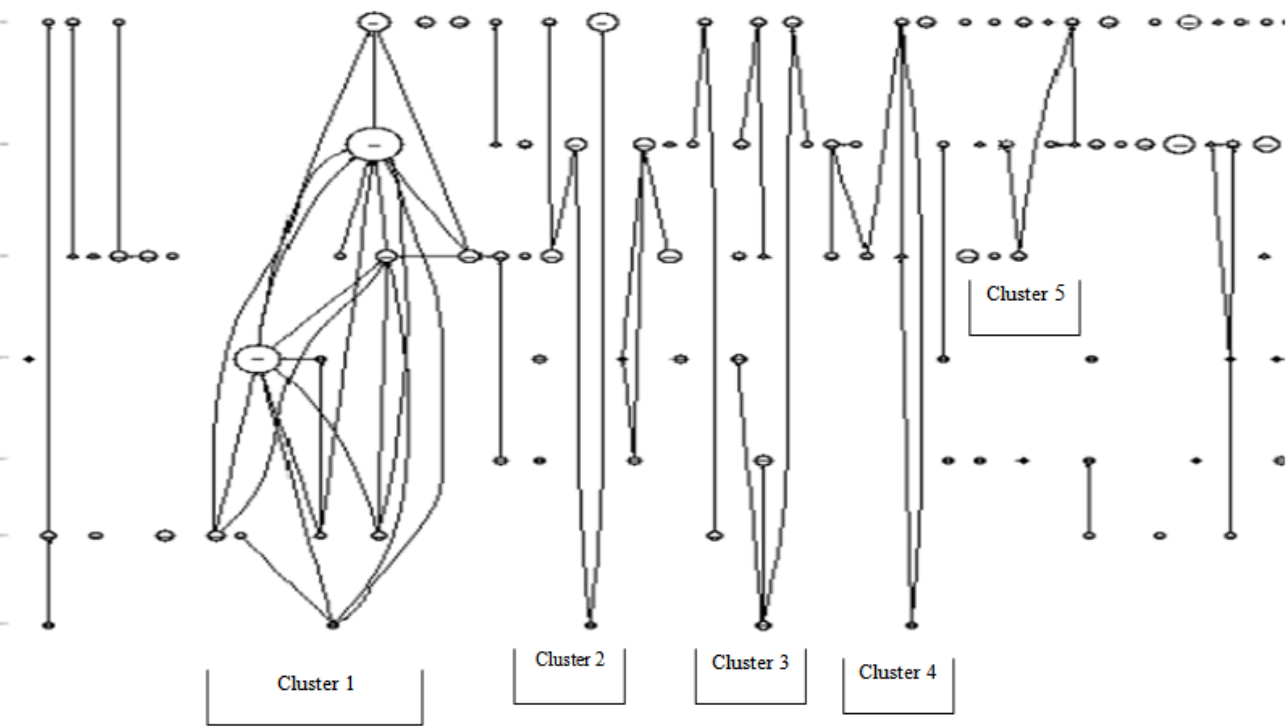

F.ig 3. Mapping of diabetes mellitus based on GCS index during 2007-2013

Turkish authors. The record number 23 had the highest local (16) and world (125) citations. The main article in this cluster belonged to Dogru, Teoman, which was titled as "Plasma visfatin levels in patients with newly diagnosed and untreated Type 2 diabetes mellitus and impaired glucose tolerance"; the article was published in the Diabetes Research and Clinical Practice journal (Fig. 2).

To do scientific mapping, 250 top documents were selected based on TGCS citations. This map consisted of 5 clusters.

The first cluster was formed during 2007-2013and included 14 articles. Evaluation of the articles revealed that the main and high citation records of this cluster were the record number 1093 (GCS=1076) and $893(\mathrm{GCS}=43)$. They were written by authors from the USA, Israel, Ireland, Thailand, Sweden, and China. The title of the article was as follows: Hyperglycemia and adverse pregnancy outcomes, which was published in 2008.

The second main article of this cluster was the article of Metzger, Boyd E, which was published in Diabetes Care in 2010. This article (International Association of Diabetes and Pregnancy Study Groups recommendations on the diagnosis and classification of hyperglycemia in Pregnancy) had 745 global citations. Gestational diabetes and its prevalence and complications were the main issues of this cluster.

The second cluster with 5 articles was formed during 2007- 2013, which was the result of collaboration of Israeli authors. The most effective article in this cluster (number 523), with 336 global citations, belonged to Nir, Tomer, which was titled as "Recovery from diabetes in mice by beta cell regeneration". The other highly cited articles of the cluster had the record number 970 $(\mathrm{GCS}=172)$ and 1796 (GCS=164). Insulin resistance, hyperglycemia, and Beta-Cell were the main issues of this cluster.

The third cluster was formed with 5 articles during 2007- 2013. The record number 329 had the highest world citation (126). The most important article in this cluster belonged to Phillip, Moshe, et al. and was published in Diabetes Care in 2007. The second main article in this 
cluster belonged to Battelino, Tadej et al. This article evaluated the effect of continuous glucose monitoring on hypoglycemia in Type 1 diabetes and had 109 global citations and was the result of collaboration of authors from Israel and Slovenia. The main issue of this cluster was Type 1 diabetes and its management.

The forth cluster was designed with 7 articles during 2007-2013. The main cluster addressed the prevalence of diabetes and impaired fasting glucose in the adult population of Iran, National Survey of Risk Factors for Non-Communicable Diseases of Iran, which was written by Alireza Esteghamati and published in Diabetes Care in 2008; this article had the highest global citation (100). The main subject of this cluster was the prevalence of diabetes and its risk factors.

The fifth cluster belonged to Iran. The article titled as "Randomized trial of intravitreal bevacizumab alone or combined with triamcinolone versus macular photocoagulation in diabetic macular edema", which was written by Masoud Soheilian and published in the Ophthalmology Journal in 2009, had 90 global citations. The other high citation articles in this cluster were 988 $(\mathrm{GCS}=64)$ and $656(\mathrm{GCS}=60)$ (Fig. 3).

\section{Discussion}

Scientists, librarians, philosophers, authorities, and publishers are interested in evaluating scientific products, mapping of the science structure in different fields, and following the last changes. Scientific texts are the main materials for this mapping (36).

With regards to the importance of medical fields, this study drew the map of diabetic disease and selected topical branches in $\mathrm{MeSH}$ using the HistCite software. Global and local indices were used to interpret the mapping. The mapping indicated an overall imaging of diabetic disease in the Middle East.

Research is focused on diabetes in many countries (1718). The higher level of diabetes publication in the Middle East belongs to Turkey, Israel, and Iran, owing to the large population size and research centers in Iran and Turkey and the strong collaboration of Israel with international researchers, especially the United states (37).

This research revealed that the trend of diabetes publishing in the Middle East is rising. Based on this research, among the Middle Eastern countries, Turkey, with 2126 records, achieved the first rank in scientific production in the field of diabetes and Iran, with 1446 records, Israel, with 1104 records, Egypt, with 607 records, and Saudi Arabia, with 547 records, achieved the next ranks, respectively.

Peykari et al. conducted a study on diabetes in the Middle East in a scientometric study. They found that among 17 Middle Eastern countries, Turkey achieved the first rank, followed by Israel and Iran (19).

With regards to the number of retrieved articles, the results of our study were not consistent with those of Peykari et al.

In 2011, Peykari showed that Iran is in the second rank with $22 \%$ scientific production (19), similar to our study.
The study of Shirshahi et al. revealed that Turkey and Iran ranked first and third, respectively, in the field of surgery, as found in this study (23).

Study of Peymani showed the trend of stem cell studies. According to this study, Turkey, with $29.56 \%$ and Iran, with $14.25 \%$, ranked first and second, respectively. These results were similar to the present study (20).

Shahram et al. (21) evaluated the scientometric analysis and mapping of scientific articles on Behcet's disease. Among the Middle Eastern countries, Turkey, with $26.55 \%$, Israel, with $2.87 \%$, and Iran, with $2.33 \%$, were in the first, second, and third ranks. However, the results of this study were not in line with those of the present study regarding Iran's rank.

In this research, Turkey and Iran achieved the first and second ranks, respectively, in the production of science in the field of diabetes. According to these results, Turkey has a significant role in research in the field of diabetes in the Middle East. Thus, scientific communication with researchers of this country will promote the science of endocrinology and metabolism and will also improve diabetes control in this region.

This study revealed that considering the number of published records (417) and local citations (295), Tehran University of Medical Sciences (TUMS) achieved the first rank in scientific production on diabetes in the Middle East.

With regards to global citations, Tel Aviv University achieved the first rank with 6713 citations.

According to the report from Scimago Institute in the field of ranking of all Middle East universities in 2011, which was done based on Scopus data, Tel Aviv achieved the first rank in the region and the 12th rank in the world, and Tehran University of Medical Sciences achieved the 7 th rank in the region and the 464th rank in the world. These results show the superiority of these universities in the world and the region and confirm our results regarding the significant role of these universities in the Middle East in the field of diabetes during 2007-2013.

In addition, Jan-Mohammadi et al. evaluated scientific productions in Iran in the field of orthopedics in 2012. They found that Tehran University of Medical Sciences produced the largest number of scientific documents in the field (26).

Hodhodinezhad et al. assessed the scientific production and mapping of Iranian researchers in the field of traditional medicine. Their results showed that Tehran University of Medical Sciences produced the largest number of records (24). This result is not shown in our study, however, the results are similar to ours with respect to local citations. Tehran University of Medical Sciences has the first rank considering the number of local citations and publications, so using these publications can promote diabetes management.

According to the results, the Journal of Diabetes Research and Clinical Practice had the largest number of issued documents in the field of diabetes (144 records). Based on the global citations, Diabetes Care Journal ranked first with 3928 citations, while based on local citations, Diabetes Research and Clinical Practice ranked 
first with 185 citations. Hodhodinezhad evaluated the scientific production of Iranian researchers in the field of traditional medicine (24). They found that the Journal of Ethnopharmacology published the largest number of documents in this field.

Moreover, Krishnamoorthy et al. evaluated the bibliometric analysis of the literature on diabetes. The results showed that Diabetes Care Journal, with 4729 records, was in the first place (17); this result was consistent with that of the present study.

Scientific journals are the most important official channels for exchange of information. Thus, recognition of important and valid journals in each topical field is of paramount importance.

In addition, Makizadeh and Osareh addressed the mapping of medical ethics in 2011. Their results showed that Journal of Medical Ethics has published the largest number of scientific products and known as the best journal in this field (27).

The results showed that most scientific products in the field of diabetes were in the form of article.

Our results about the format of documents are similar to those of Namdar (25). Furthermore, in the study of Makizadeh and Osareh (27), it was found that the most published scientific products are in the form of articles.

The results of the research show that articles were the most commonly used format for exchanging scientific information as shown in our research.

In Shahram (21) study, original articles, with 55.9\%, and meeting abstracts, with $16.7 \%$, were in the first and second ranks, respectively, which are in line with this research. Considering the publication type, the results were as same as Krishnamorty's (17). The results regarding the fact that articles with 667 records are in the first rank and meeting abstracts with 111 records are in the second rank were consistent with Arunachslam's study (18).

Most of the products were articles, and this may be due to the fact that the information of articles is newer than other forms of publications. Thus, the researchers publish their newest scientific products in the form of article. Furthermore, researchers and authors rely on institutions of higher education and universities and need research score for promotion of their academic ranking. The score of publication of an article in a journal is higher than other formats, so researchers are interested in publishing their works in the form of article.

Results showed that most documents are published in English; 97\% of documents were in English as per the study conducted by Makizadeh (27), Shahram (21), and Arunachalam (18).

This indicates a significant difference in the dominance of English language as a scientific language in the WoS.

Also, the results revealed that scientific products of Iranian researchers in the field of diabetes and their global and local citations are satisfactory.

Iran achieved the second rank in diabetes research during the study period. The scientific map of endocrinology consisted of 7 important clusters in the LCS and 5 in the GCS, which were the results of efforts of
Middle Eastern researchers. Regarding the global increase in the prevalence of diabetes and the Middle East, the results of our study can be useful for the researchers.

Furthermore, the main clusters were concerned with the prevalence of diabetes and its complications, Type 1 diabetes, and gestational diabetes. These results can be applied by health care providers to determine the priorities for preventive and therapeutic interventions. In addition, these findings will help governments and policy-makers to reorient priorities of funding supports for both research and health care policies.

\section{Conclusion}

In conclusion, the trend of diabetes publication in the Middle East is rising. The highest number of scientific outputs belongs to turkey, Iran, and Israel, followed by other counties, which considerably had a lower rate of production. Moreover, Iranian research in the field of diabetes is adequately desirable, causing the country to be placed in a good position. In addition, the clusters showed that the prevalence of diabetes and its complications, Type 1 diabetes, and gestational diabetes are the focus of attention of the researchers. The growth of the literature in the field of diabetes in the Middle-East and Iran indicates the growing incidence rate of diabetes and its complications in this region. This result will encourage the health care providers and policy- makers to set priorities for better management and prevention of diabetes and its complications using a multidisciplinary approach. However, other important aspects of diabetes should also be studied by the investigators.

\section{Conflict of interests}

The authors declare that they have no competing interests.

\section{References}

1. Lozano R, Naghavi M, Foreman K, Lim S, Shibuya K, Aboyans V, et al. Global and regional mortality from 235 causes of death for 20 age groups in 1990 and 2010: a systematic analysis for the Global Burden of Disease Study 2010. Lancet. 2012;380:2095-128.

2. Murray CJ, Vos T, Lozano R, Naghavi M, Flaxman AD, Michaud C, et al. Disability-adjusted life years (DALYs) for 291 diseases and injuries in 21 regions, 1990-2010: a systematic analysis for the Global Burden of Disease Study 2010. Lancet. 2012;380:2197-223.

3. IDF Diabetes Atlas Sixth Edition Poster Update 2014. Available from: https://www.idf.org/diabetesatlas/update-2014?language $=$ en

4. International Diabetes Federation (IDF) [Internet]. Country estimates table 2011. IDF diabetes atlas. 6th ed. 2012. Available from: http://www.idf.org/

5. Norozi chakoli A, Hasanzadeh M, Noormohammadi H, Etemadifard A. Scientific Products of Iran in ISI during the 15 Years between 1993 -2007. IJISM. 2010;8(2):11-27. [Persian]

6. Ebrahimi S, Hayati Z. The quantity and quality of knowledge produced in Iranian universities. Quarterly Journal of New Thoughts on Education. 2008; 4: 105-126. [Persian]

7. Garfield E. When to cite. Lib Q. 1996;66:449-58.

8. Escudero Gómez CMillán Santos I, Posada de la Paz M. Analysis of Spanish scientific production in rare diseases: 1990-2000. Med Clin. 2005;125:329-32.

9. Vitzthum K, Mache S, Quarcoo D, Scutaru D, Groneberg DA, Schöffel N. Scoliosis: density-equalizing mapping and scientometric analysis. Scoliosis. 2009;4:15

10. BaIa A, Gupta B. mapping of Indian Neuroscience Research: A scientometric analysis of research output during 1999-2008. Neurol India. 2010;58(1):35-41. 
11. Li LL, Ding GH, Feng N, Wang MH, Ho YS. Global stem cell research trend: Bibliometric analysis as a tool for mapping of trends from 1991 to 2006. Scientometrics. 2009;80(1):39-58.

12. Patra SK, Chand P. HIV/AIDS research in India: A bibliometric study. Libr Inf Sci Res. 2007;29(1):124-134.

13. Yang S, Needleman H, Niederman R. A bibliometric analysis of the pediatric dental literature in MEDLINE. Pediatr Dent. 2001; 23:415-8.

14. Ramos JM, Gutierrez F, Royo G. Scientific production in microbiology and affinity areas in Spain during 1990-2002. Enferm Infecc Microbiol Clin. 2005;23:406-14.

15. Signore A, Annovazzi A. Scientific production and impact of nuclear medicine in Europe: how do we publish? Eur J Nucl Med Mol Imag. 2004;31:882-6.

16. Stegmann J, Grohmann G. Cooperation bibliogram of bird flu, 2006. In International workshop on webometrics, Informetrics and scientometrics and seventh COLLENT meeting, Nancy: France; 2006.

17. Krishnamoorthy G, Ramakrishnan J, Devi S. Bibliometric analysis of literature on diabetes (1995-2004). ALIS. 2009;56:150-155.

18. Arunachalam S, Gunasekaran S. Diabetes research in India and China today: From literature-based mapping to health-care policy. Curr Sci. 2002;82:1086-1097.

19. Peykari N, Djalalinia S, Kasaeian A, Naderimagham S, Hasannia T, Larijani B, et al. Diabetes research in Middle East countries; a scientometrics study from 1990 to 2012. J Res Med Sci. 2015;20(3): 253-262.

20. Peymani P, Zahiri Z, Heydari ST, Khoshsima M, Lankarani KB. Stem cell research trends: bibliometric analysis as a tool for mapping trends from 1995 to 2010. World J Med Sci. 2012;7 (3):159-162.

21. Shahram F, Jamshidi AR, Hirbod-Mobarakeh A, Habibi G, Mardani A, Ghaemi M. Scientometric analysis and mapping of scientific articles on Behcet's disease. Int J Rheum Dis. 2013;16(2):185-92.

22. Hamdipour A, Osareh F. Scientometric Study and Visualization of Multiple Sclerosis-Related Publications during 1999-2008. Health Inf Manage. 2011;8:661. [Persian]

23. Shirshahi S, Osareh F, Haidari Gh, loni N. Mapping the structure of surgery discipline in Science Citation Index. Health Inf Manage. 2015; 11:83. [Persian]

24. Hodhodinezhad N, Zahedi Anaraki R, Ashrafi Rizi H. The Scientific Production and Scientific Mapping of Iranian Researchers in Traditional Medicine during 1990-2011 in Web of Science. Health Inf Manag. 2012; 9: 524. [Persian]

25. Namdar L, Salajegheh M, Osareh F. Tracing the Scientific Status of Researchers' Outputs in the Field of Parasitology as Reflected in Science Citation Databases. J Stri Devel Med Edu. 2013;10:13-26. [Persian]

26. Janmohammadi N, Yaminfirooz M, Nooshinfard F. Iran's Scientific Production in Orthopaedic Field: A Scientometric Study. Iranian $J$ Orthoped Surg. 2012; 10 (2):81-86.

27. Makizadeh F, Osareh F. Citation analysis and algorithmic histography of medical ethics in Web of Science in 1990-2008. Iranian J Med Ethic History Med. 2011;4(5):65-77.

28. Thelwall M. Bibliometrics to webometrics. J Inf Sci. 2008;34(4): 605-21.

29. Garfield E, Pudovkin AL, Istomin VS. Why Do We Need Algorithmic Historiography? JASIST. 2003;54(5):400-412.

30. Garfield E, Paris SW, Stock WG. Histcite: A software tool for informatics analysis of citation linkage. Information Wissenschaft und Praxis. 2006;57(6):391-400.

31. Garfield E, Pudovkin AL, Istomin VS. Mapping Output of Topical Searche in web of knowledge and case of waston-Crick. Info Tech Lib. 2003;22(4):183-7.

32. Lucio-Arias D, Leydesdorff L. Main-path analysis and pathdependent transitions in HistCite ${ }^{\mathrm{TM}}$-based historiograms. J Am Soc Info Sci Technol. 2009; 59(12):1-28.

33. Guz AN, Rushchitsky JJ. Scopus: A system for the evaluation of scientific journals. Int Appl Mech. 2009;45(4):351-362.

34. Alexander JL, Aleixandre JL, Bolanos-Pizarro M, AleixandreBenavent R. Mapping the scientific research in organic farming: a bibliometric review. Scientometrics. 2015;105(1):295-309.

35. List of Middle East Countries by Population. Available from: www.worldatlas.com

36. Paul RJ. Visualizing a Knowledge Domain's Intellectual Structure [Online]. 2001; Available from: URL: http://www.mendeley.com/ research/visualizing-a-knowledge-domains-intellectual-structure/

37. Harande Y. Exploring the literature of diabetes in Nigeria: A 